\title{
Many-body Interactions in a Sample of Ultracold Rydberg Atoms with Varying Dimensions and Densities
}

Thomas J. Carroll

Ursinus College, tcarroll@ursinus.edu

Shubha Sunder

Michael W. Noel

Follow this and additional works at: https://digitalcommons.ursinus.edu/physics_astro_fac

Part of the Atomic, Molecular and Optical Physics Commons

Click here to let us know how access to this document benefits you.

\section{Recommended Citation}

Carroll, Thomas J.; Sunder, Shubha; and Noel, Michael W., "Many-body Interactions in a Sample of Ultracold Rydberg Atoms with Varying Dimensions and Densities" (2006). Physics and Astronomy Faculty Publications. 2.

https://digitalcommons.ursinus.edu/physics_astro_fac/2 


\title{
Many-body interactions in a sample of ultracold Rydberg atoms with varying dimensions and densities
}

\author{
Thomas J. Carroll, Shubha Sunder, and Michael W. Noel \\ Department of Physics, Bryn Mawr College, Bryn Mawr, Pennsylvania 19010, USA
}

(Received 31 May 2005; published 27 March 2006)

\begin{abstract}
Ultracold highly excited atoms in a magneto-optical trap (MOT) are strongly coupled by the dipole-dipole interaction. We have investigated the importance of many-body effects by controlling the dimensionality and density of the excited sample. We excited three different cylindrical volumes of atoms in the MOT to Rydberg states. At small radius, where the sample is nearly one-dimensional, many-body interactions are suppressed. At larger radii, the sample becomes three-dimensional and many-body effects are apparent.
\end{abstract}

DOI: 10.1103/PhysRevA.73.032725

PACS number(s): 34.60.+z, 32.80.Pj, 03.67.Lx, 32.80.Rm

By exciting the atoms in a laser-cooled gas to weakly bound Rydberg states, they can be made to interact strongly over the several micrometer distance separating them. In many cases, the atoms can exchange energy quickly enough that they move very little during the experiment. This "frozen Rydberg gas" resembles an amorphous solid, but on a greatly magnified scale $[1,2]$. One advantage of this system over an amorphous solid is the precision with which the internal structure of the atoms as well as their spatial arrangement can be manipulated to control the interactions among atoms. In particular, the states of the atom can be shifted with a small static electric field to bring into resonance a dipole-dipole driven exchange of energy between atoms. In such a frozen gas, this energy exchange does not occur through binary collisions, but rather through the simultaneous interactions among many atoms. These many-body effects were first revealed through a broadening of the resonant energy exchange, which could not be accounted for by simply considering two-body interactions [3-5]. Further insight has been gained by tailoring the mixture of excited Rydberg states to bring additional always resonant interactions into play in a well controlled manner [6,7].

In addition to mediating the exchange of energy among atoms, the dipole-dipole interaction provides an attraction between atoms that can lead to ionizing collisions, which seed the evolution of the sample into an ultracold plasma [8-10]. Ultimately the dipole-dipole interaction between Rydberg atoms may be useful for entangling atoms and building fast quantum gates [11-14]. Thus far, studies of frozen Rydberg gasses have focused on manipulating the internal states of the excited atoms. In this paper, we focus on the control of the spatial arrangement of the atoms and its effect on the resonant exchange of energy among them. By exciting a long narrow tube of Rydberg atoms of varying density and aspect ratio, we are able to effectively vary the dimensionality of the system between one and three. In the one-dimensional case many-body effects are suppressed, while in the threedimensional sample they become apparent.

We begin by collecting a sample of rubidium atoms in a magneto-optical trap (MOT). A pulsed blue laser beam is then focused through the trap to excite a long narrow tube of Rydberg states. This excitation is done in the presence of a static electric field, which shifts the states of the atoms so that they may resonantly exchange energy. After a few microseconds of interaction time, we field ionize the atoms and measure the fraction of excited atoms that have undergone a resonant energy exchange. We vary the number of excited atoms by changing the intensity of the blue laser beam and vary the aspect ratio of the tube by adjusting the diameter of the blue laser beam.

We trap ${ }^{85} \mathrm{Rb}$ atoms using diode lasers in a standard MOT [15-17]. The typical trap for this experiment had a diameter of $350 \mu \mathrm{m}$ (full width at half maximum) and contained about $8 \times 10^{5}$ atoms at a temperature of approximately $300 \mu \mathrm{K}$. The MOT is centered in a configuration of four parallel field wires, arranged at the corners of a square of side $1.27 \mathrm{~cm}$.

To produce Rydberg atoms, the ${ }^{85} \mathrm{Rb}$ atoms are excited to the $31 d$ level from the $5 p_{3 / 2}$ state using a $480 \mathrm{~nm}$ pulsed dye laser (Coumarin-480) pumped with a frequency tripled $\mathrm{Nd}$ :YAG laser running at $20 \mathrm{~Hz}$. The beam passes through a circular, continuously variable neutral density filter which varies from an optical density of 0 to 3 . A doublet-meniscus lens combination is used to focus the beam into the trap. An iris is placed before the lens to control the diameter of the beam and thus the beam waist at the focus. Knife-edge measurements of the radius of the beam at the focus yield $6.5,11$, and $19 \mu \mathrm{m}$ for input beam diameters of 5.97, 3.05, and $1.65 \mathrm{~mm}$, respectively. The focus of the beam is carefully overlapped with the MOT. This is done by translating the lens assembly along the laser's propagation direction. With the laser power set high enough to saturate the Rydberg excitation, the position that minimizes the total electron signal also minimizes the excited atom volume.

By applying voltages to the field wires, a static electric field is produced at the trap. At various electric field strengths, there are Stark states equally spaced in energy above and below the excited $d$ state, where a resonant energy exchange can occur,

$$
31 d+31 d \rightarrow 33 p+(n=29),
$$

where $(n=29)$ refers to manifold states. In Fig. 1(a), we plot the fraction of the atoms initially excited to the $31 d$ state that end up in the $33 p$ state as the static electric field is varied, revealing a multitude of field-tuned resonances. Figure 1(b) shows a Stark map for the region around 31d along with a 


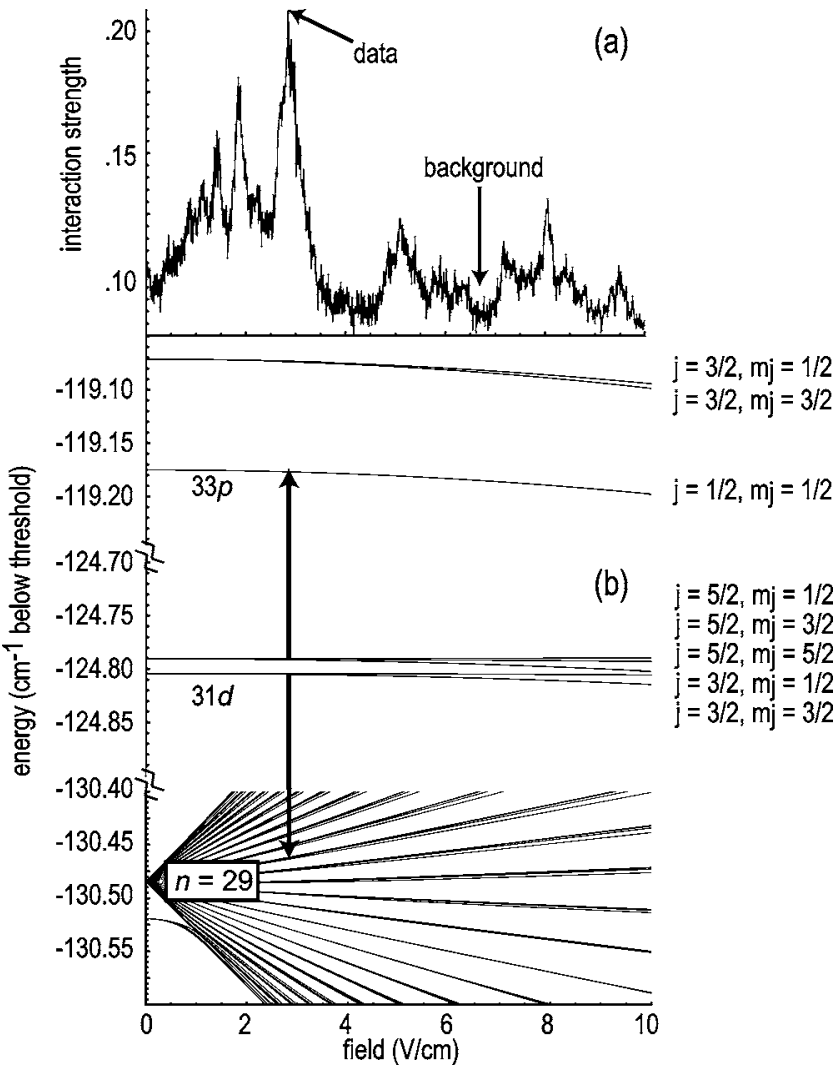

FIG. 1. Stark map of the $31 d$ states, $33 p$ states, and $n=29$ manifold states that contribute to the resonances of Eq. (1). The example resonance shown in (b) depicts two interacting atoms that have been excited to $31 d$ states. Via the dipole-dipole interaction, one atom is further excited to a $33 p$ while the other falls to a manifold state. Both the $33 p$ and $31 d$ states are detected using selective field ionization. Also shown in (a) is a scan over electric field for a fixed laser intensity. The strong resonance, labeled data, is where we collected our data. The area of little interaction, labeled background, is where we collected the background.

typical resonance of the type described by Eq. (1), which are dominant in our region of interest. Each of the broad peaks in Fig. 1(a) is composed of many overlapping resonances due to the large number of $j$ and $m_{j}$ values for both the initial and final states that can exchange energy over a very narrow range of field-tuned resonances. The energy exchange is mediated by this interaction,

$$
V=\frac{\boldsymbol{\mu}_{\mathbf{1}} \cdot \boldsymbol{\mu}_{\mathbf{2}}-3\left(\mu_{1} \cdot \hat{\mathbf{R}}\right)\left(\boldsymbol{\mu}_{\mathbf{2}} \cdot \hat{\mathbf{R}}\right)}{R^{3}},
$$

where $\boldsymbol{\mu}_{1}$ and $\boldsymbol{\mu}_{2}$ are the $31 d \rightarrow 33 p$ and $31 d \rightarrow(n=29)$ electric dipole matrix elements and $\mathbf{R}$ is the separation vector connecting the pair of atoms [18].

At the end of a $3 \mu$ s interaction time, a high voltage field ionization pulse is applied to the two field wires furthest from the detector. The pulse amplitude is adjusted to ionize the initial $31 d$ state along with any atoms that have been transferred to the $33 p$ state. The field ionization pulse accelerates the ionized electrons toward a chevron microchannel plate assembly where they are detected. The field ionization

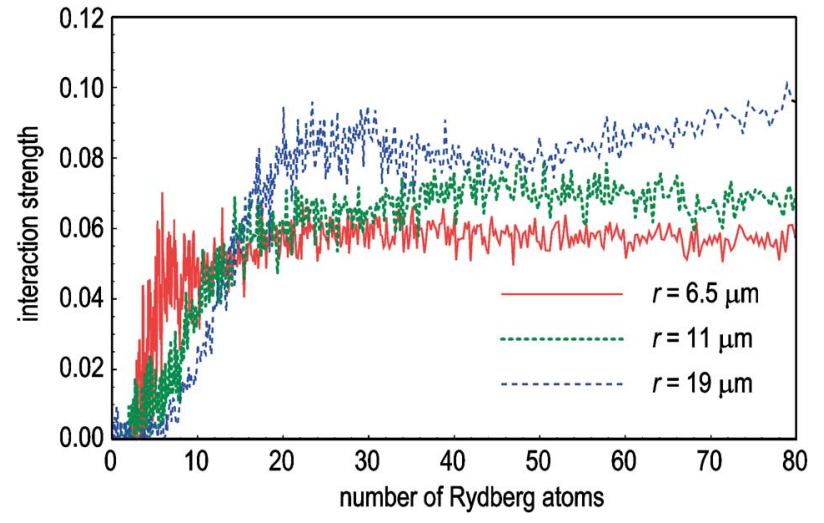

FIG. 2. (Color online) Data taken at three different excited volumes. Even though the density is higher in the smaller, onedimensional volume, the interaction is stronger in the sparser but more three-dimensional volumes.

pulse has a rise time of $1.5 \mu$ s and provides sufficient energy resolution to time resolve the $31 d$ signal from the $33 p$ signal, but not to resolve the $j$ or $m_{j}$ sublevels of these states. Atoms that end up in the $n=29$ manifold are not detected.

The time-resolved electron signal is collected as the intensity of the beam is scanned over many shots of the laser. The fraction of atoms that interacts is found by integrating the first peak in the time-resolved signal, which is associated with the $33 p$ state, and normalizing by the total signal. The total signal is calculated by adding the $31 d$ signal to twice the $33 p$ signal since the interaction of Eq. (1) leaves one atom in the $n=29$ manifold, which is not detected, for each atom that ends up in the $33 p$ state. The electric field is tuned to a strong resonance at about $3 \mathrm{~V} / \mathrm{cm}$ to collect the data [see Fig. 1(a)]. To measure the background, due primarily to black-body transitions, similar data are collected with the electric field tuned to an area of very weak interaction near $6 \mathrm{~V} / \mathrm{cm}$. The data are binned by the total signal, which is proportional to the number of Rydberg atoms in the sample, and the background is subtracted. The result is a plot of interaction strength (as measured by the fraction that interact) versus number of Rydberg atoms, as shown in Fig. 2.

The calibration from total signal to number of atoms in Fig. 2 was performed by multiplying the measured volume of the excited sample by the density of the trap to yield the number of atoms available for excitation. This was then multiplied by the excitation probability to yield the number of Rydberg atoms, to within about a factor of 2 .

The general shape of the three curves in Fig. 2 is a rise in the fraction of atoms in the $33 p$ state followed by a saturation at some level. When there are only a few atoms in a particular volume, the typical spacing between them will lead to a small interaction strength [due to the $1 / R^{3}$ scaling in Eq. (1)] and thus a small fraction in the $33 p$ state. As the number increases, and the typical separation decreases, more atoms will exchange energy resulting in a larger $33 p$ fraction. When the density gets large enough that the Rabi period is less than the $3 \mu$ s interaction time, the fraction in the $33 p$ state will begin to saturate.

Although the three curves in Fig. 2 have the same general shape, a comparison of the three reveals a clear dependence 

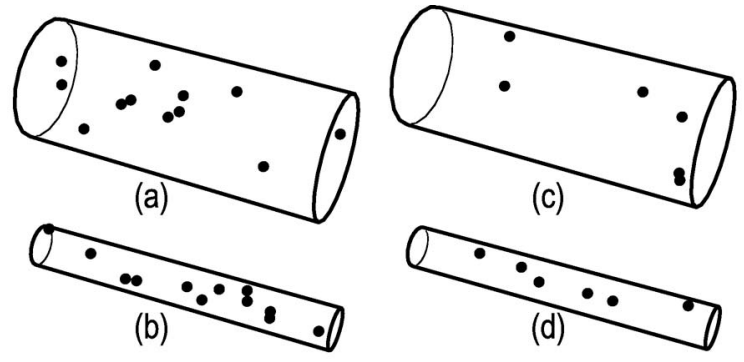

(c)
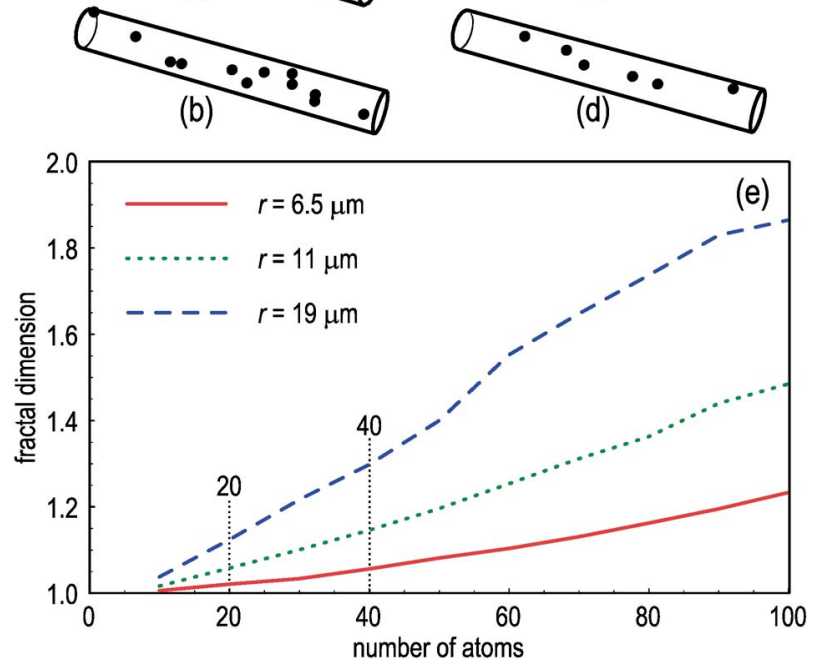

FIG. 3. (Color online) Shown above, in (a)-(d), are $100-\mu \mathrm{m}$-long sample volumes of radii 6.5 and $20 \mu \mathrm{m}$ with randomly placed atoms. Volumes (a) and (b) correspond to a $350 \mu \mathrm{m}$ volume with 40 atoms, while volumes (c) and (d) correspond to such a volume with 20 atoms. In (e) we plot the fractal dimension for $350 \mu \mathrm{m}$ length volumes of different radii. Note that the $6.5 \mu \mathrm{m}$ radius volume remains nearly one-dimensional even to relatively high number.

on the dimension of the excitation volume. At low numbers of Rydberg atoms, the smallest volume has the highest density and therefore the largest interaction strength. In Fig. 2, this leads to the smallest volume having a larger initial slope. As the number of Rydberg atoms increases, the interaction strength for the larger volumes eventually surpasses that of the smallest volume even though they still have lower densities. This crossing is seen in Fig. 2 when there are about 15 atoms in each volume. With 15 atoms in the smallest volume, the average spacing between an atom and its nearest neighbor is about $13 \mu \mathrm{m}$. In the largest volume, this spacing is roughly $19 \mu \mathrm{m}$. We attribute this phenomenon to the presence of many-body interactions in the larger volume samples.

Since the smallest beam diameter is on the order of the average spacing of the Rydberg atoms for the highest numbers excited, this volume is nearly one-dimensional for a wide range of numbers of atoms. The larger volumes are successively more three-dimensional, as seen in Figs. 3(a)-3(d). In the one-dimensional case, each atom will typically have only two nearest neighbors and we expect the interaction to be dominated by two-body interactions. For the larger volumes, each atom has access to more neighbors and the interaction strength is enhanced by many-body interactions. This enhancement was seen in the first spectroscopic measurements of resonant energy exchange among cold Rydberg atoms $[3,4]$. In these experiments, broad resonances were observed involving a much larger fraction of atoms than could be accounted for by the simple two-body energy exchange between close pairs of atoms. Further systematic studies have verified the importance of an always resonant exchange resulting in a diffusion of energy away from pairs of close atoms that rapidly exchange energy through the field tuned interaction [5]. The combined effect of the always resonant and field tuned energy exchange processes is the formation of broad energy bands similar to those in solidstate systems [6]. Our data reveal that these many-body effects can be reduced by restricting the dimensionality of the system and thus the pathways allowed for this diffusion of energy exchange.

To quantify the dimensionality of our sample, the fractal dimension for the different volumes has been calculated $[19,20]$ and is shown in Fig. 3(e). Many sets of randomly distributed atoms are generated for each volume. Successively smaller measurement scales are then used to find the total length of the sample (by traversing from one atom to the next). The fractal dimension is estimated by the parameter $D$ in

$$
\ln [L(s)]=(1-D) \ln (s)+b,
$$

where $L(s)$ is the length measured with scale $s$, and $b$ is a scale factor. If all of the atoms lie in nearly a straight line, each scale will measure about the same length and the calculated fractal dimension will be close to 1 . However, if the atoms are more widely distributed, smaller scales will be able to measure smaller features in the "line" of atoms and the measured length will increase. This results in a larger calculated fractal dimension. As the number of atoms in each volume increases, the smallest volume remains nearly onedimensional, while the fractal dimension of the largest volume rises rapidly.

A simulation code was developed to model the data $[21,22]$. We approximate our excited sample by a cylindrical volume that is randomly filled with stationary atoms. Atoms are allowed to interact via Eq. (1) and by the following always resonant interactions:

$$
\begin{gathered}
31 d+33 p \rightarrow 33 p+31 d \\
\text { and } \\
31 d+(n=29) \rightarrow(n=29)+31 d .
\end{gathered}
$$

Spin effects and the angular portion of the interaction are ignored, with the interaction approximated simply as $\mu \mu^{\prime} / R^{3}$, where $\mu \mu^{\prime}$ could be $\mu_{1} \mu_{2}, \mu_{1}^{2}$, or $\mu_{2}^{2}$.

Each atom can be in one of three states: $33 p, 31 d$, or an $n=29$ manifold state. All product states for a group of atoms are enumerated and the Hamiltonian matrix is filled based on the interactions of Eq. (1), Eq. (4a), and Eq. (4b). For small numbers of atoms, this leads to a very large number of possible states, even when states inaccessible from our initial state are ignored (such as the state with all atoms in the $31 p$ state). For nine atoms there are 3139 states, while for ten atoms there are 8953 . Thus, we are limited to simulating groups of nine atoms with the memory currently available in our computer cluster. To achieve higher atom numbers in our 


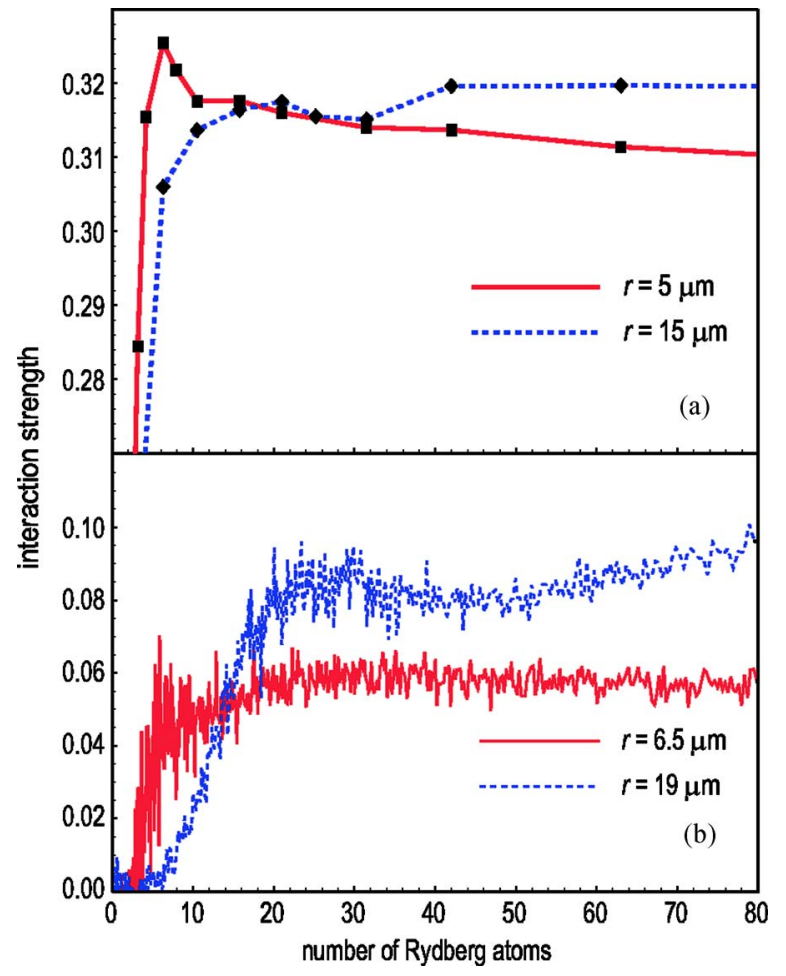

FIG. 4. (Color online) Results of the simulations with nine atoms are shown in (a) at $5 \mu$ s for two volumes of length $350 \mu \mathrm{m}$. The behavior of the $5 \mu \mathrm{m}$ and the $15 \mu \mathrm{m}$ volumes reproduces the features of the data shown in (b).

simulations, we simulate subvolumes with the same radius but a shorter length and use those to build up the full volume.

The time evolution of the atoms is simulated for two different total volumes, both of length $350 \mu \mathrm{m}$, with radii of 5 and $15 \mu \mathrm{m}$. While the resonance peak at which we collect data is composed of many superimposed interactions, we chose one representative interaction for the simulation. To reduce edge effects we calculate the probability that an atom placed centrally in each subvolume makes a transition to the experimentally measured $33 p$ state.

The simulation results shown in Fig. 4(a) for an interaction time of $5 \mu$ s reproduce the major features of our data shown Fig. 4(b). The interaction strength for the larger volume rises more slowly than that for the narrow tube and then levels off briefly while surpassing the narrow tube interaction strength. While the simulation succeeds on this level, there are some differences. To simulate larger numbers of atoms in this volume, it becomes necessary to use smaller and smaller subvolumes which no longer faithfully represent the experimental geometry. This is not as much of an issue for the narrow tube, as the subvolumes can be made significantly smaller. Perhaps the biggest discrepancy between our data and simulation is in the level at which the signal saturates for large numbers of Rydberg atoms. Our simulation models each atom as a simple three-state system while a variety of initial and final states can exchange energy in our experiment. A mixture of $j=3 / 2$ and 5/2 states are excited by our dye laser. Some neighboring atoms will be in states for which there is no resonant energy exchange or it is very weak. These atoms contribute to the total signal but not to the fraction that interact.

The other important discrepancy is that the large volume signal in our data rises more slowly than in our simulation. A contributing factor to this difference is almost certainly the angular dependence of the dipole-dipole interaction [23], which we do not model. In particular, the contribution to resonant energy exchange in the large volume sample due to many neighbors is reduced along certain directions due to the angular dependence of the interaction.

In summary, we have studied the resonant exchange of energy among atoms as the sample's dimensionality is varied. We observe a stronger interaction in the threedimensional case over the one-dimensional case when the two volumes are filled with more than 15 atoms even though the average distance between atoms is larger in the former case. We attribute this enhancement to many-body effects in the three-dimensional sample. These observations are in good qualitative agreement with a simulation that includes interactions among up to nine atoms.

This work was supported by the National Science Foundation under Grant No. 0134676. Funding for the 48-node Beowulf cluster on which the calculations were run was provided by Bryn Mawr College.
[1] J. S. Frasier, V. Celli, and T. Blum, Phys. Rev. A 59, 4358 (1999).

[2] F. Robicheaux, J. V. Hernández, T. Topçu, and L. D. Noordam, Phys. Rev. A 70, 042703 (2004).

[3] W. R. Anderson, J. R. Veale, and T. F. Gallagher, Phys. Rev. Lett. 80, 249 (1998).

[4] I. Mourachko, D. Comparat, F. de Tomasi, A. Fioretti, P. Nosbaum, V. M. Akulin, and P. Pillet, Phys. Rev. Lett. 80, 253 (1998).

[5] W. R. Anderson, M. P. Robinson, J. D. D. Martin, and T. F. Gallagher, Phys. Rev. A 65, 063404 (2002).

[6] I. Mourachko, W. Li, and T. F. Gallagher, Phys. Rev. A 70, 031401(R) (2004).
[7] K. Afrousheh, P. Bohlouli-Zanjani, D. Vagale, A. Mugford, M. Fedorov, and J. D. D. Martin, Phys. Rev. Lett. 93, 233001 (2004).

[8] W. Li, P. J. Tanner, and T. F. Gallagher, Phys. Rev. Lett. 94, 173001 (2005).

[9] M. P. Robinson, B. L. Tolra, M. W. Noel, T. F. Gallagher, and P. Pillet, Phys. Rev. Lett. 85, 4466 (2004).

[10] S. K. Dutta, D. Feldbaum, A. Walz-Flannigan, J. Guest, and G. Raithel, Phys. Rev. Lett. 86, 3993 (2001).

[11] D. Jaksch, J. I. Cirac, P. Zoller, S. L. Rolston, R. Côté, and M. D. Lukin, Phys. Rev. Lett. 85, 2208 (2000).

[12] M. D. Lukin, M. Fleischhauer, R. Cote, L. M. Duan, D. Jaksch, J. I. Cirac, and P. Zoller, Phys. Rev. Lett. 87, 037901 
(2001).

[13] I. E. Protsenko, G. Reymond, N. Schlosser, and P. Grangier, Phys. Rev. A 65, 052301 (2002).

[14] M. S. Safronova, C. J. Williams, and C. W. Clark, Phys. Rev. A 67, 040303(R) (2003).

[15] E. L. Raab, M. Prentiss, A. Cable, S. Chu, and D. E. Pritchard, Phys. Rev. Lett. 59, 2631 (1987).

[16] D. W. Sesko, T. Walker, and C. Wieman, J. Opt. Soc. Am. B 8, 946 (1990).

[17] C. D. Wallace, T. P. Dinneen, K. Y. N. Tan, A. Kumarakrishnan, P. L. Gould, and J. Javanainen, J. Opt. Soc. Am. B 11, 703 (1994)

[18] T. F. Gallagher, Rydberg Atoms, Cambridge Monographs on
Atomic, Molecular, and Chemical Physics (Cambridge University Press, Cambridge, UK, 1994).

[19] L. F. Richardson, General Systems Yearbook 6, 139 (1961).

[20] B. B. Mandelbrot, The Fractal Geometry of Nature (Freeman, San Francisco, 1982).

[21] B. Heimsund, Matrix Toolkits for Java, URL http:// rs.cipr.uib.no/mtj/.

[22] W. H. Press, B. P. Flannery, S. A. Teukolsky, and W. T. Vetterling, Numerical Recipes in C: The Art of Scientific Computing (Cambridge University Press, Cambridge, UK, 1988).

[23] T. J. Carroll, K. Claringbould, A. Goodsell, M. J. Lim, and M. W. Noel, Phys. Rev. Lett. 93, 153001 (2004). 